\title{
Weathering the storm: How can thoracic surgery training programs meet the new challenges in the era of less-invasive technologies?
}

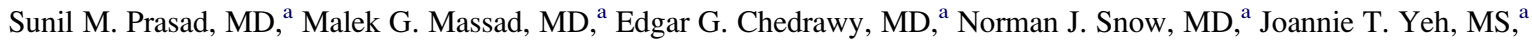 \\ Himalaya Lele, MD, ${ }^{a}$ Ahmed Tarakji, MD, ${ }^{a}$ Hersh S. Maniar, MD,${ }^{b}$ Heather Herren, RN, MPH, and William A. Gay, MD
}

Objective: The introduction of new technologies has shifted some resident index procedures to nonsurgical specialists. We examined the operative case volume of thoracic surgery residents during the last 6 years to objectively identify changes and trends.

Methods: Program and resident data from 2002 to 2007 were entered into a database and analyzed. Program match information was obtained from the National Resident Matching Program. Resident operative experience and board examination results were obtained from the American Board of Thoracic Surgery.

Results: A total of 795 residents qualified for the written American Board of Thoracic Surgery examination; 627 residents graduated from 2-year programs, and 168 residents graduated from 3-year programs. The total number of resident cases was higher in 3-year programs compared with 2-year programs in all 10 index categories studied $(P<.01)$. The total volume of cases has not significantly increased in 2 -year programs. The volume of coronary artery bypass graft surgeries decreased in every resident program model studied. The volume of general thoracic cases increased in all program models. Two-year, 2-resident programs had the lowest volume in 5 of the 10 categories, reaching significance in 3 categories. The written board pass rate was lower among 2 -year programs than among 3 -year programs ( $86 \%$ vs $95 \%$, respectively, $P=.003)$.

Conclusion: Training programs have so far weathered the storm by maintaining index volume with a new case mix, but significant trends in revascularization procedures are concerning. This study indicates a significant advantage in case volume and board pass rates among 3 -year programs. Thoracic residency programs should be reorganized so that the number of residents does not exceed the capacity of the program to provide a meaningful experience.

\section{Supplemental material is available online.}

The Residency Review Committee for Thoracic Surgery (RRC-TS) and the American Board of Thoracic Surgery (ABTS) have established requisite numbers of "index" operative procedures as one measure of the adequacy of a resident's education experience. The emergence of new, less-invasive technologies has affected the number of certain operative procedures available as part of resident education. ${ }^{1-5}$ This impact also has been felt by the practicing cardiothoracic surgeon. In addition, changes in thoracic surgery residencies, such as the 80-hour work week and the declining interest among general surgery residents to pursue further training in thoracic surgery, have stimulated us to investigate whether these issues may have adversely affected the resident operative volume and the pass rate on the written and oral examinations administered by the ABTS.

\footnotetext{
From the Divisions of Cardiothoracic Surgery, University of Illinois at Chicago, ${ }^{a}$ Chicago, Ill; Christiana Care Health System, ${ }^{\text {b }}$ Newark, Del; and Washington University School of Medicine, ${ }^{\mathrm{c}}$ St Louis, Mo.

Received for publication May 8, 2008; revisions received Jan 7, 2009; accepted for publication Feb 16, 2009

Address for reprints: Sunil M. Prasad, MD, Division of Cardiothoracic Surgery, The University of Illinois at Chicago, 840 S. Wood Street, CSB Suite 417 (MC 958), Chicago, IL 60612 (E-mail: smprasad@uic.edu).

J Thorac Cardiovasc Surg 2009;137:1317-26

$0022-5223 / \$ 36.00$

Copyright (c) 2009 by The American Association for Thoracic Surgery

doi:10.1016/j.jtcvs.2009.02.029
}

The purpose of this study was to examine the operative experience of residents in American Council on Graduate Medical Education (ACGME)-accredited thoracic surgery residencies during the last 6 years and to follow the performance of this group of residents through the ABTS certification process. Our specific aims were to 1) identify changing patterns in the National Resident Matching Program (NRMP) thoracic residency match, 2) document operative volume and case distribution of thoracic residents during the study period, 3) evaluate the different educational models based on size and duration of thoracic residency programs, and 4) analyze the performance of recent thoracic residents on the ABTS written and oral examinations.

\section{MATERIAL AND METHODS}

Resident match information was obtained from the NRMP. Operative experience of thoracic surgical residents who qualified for the written examination of the ABTS from 2002 to 2007 was obtained from data submitted by residents and program directors making an application to the ABTS. All identifying resident and program information was blinded to the investigators. The yearly pass/fail rates on the certifying examinations were obtained from the ABTS. This study was reviewed by the institutional review board of the University of Illinois and was approved. The data were entered into an Excel (Microsoft Corp, Redmond, WA) database for evaluation.

\section{American Board of Thoracic Surgery Index Case Categories}

Ten index case categories were identified for statistical evaluation. These categories include pulmonary resections (PULM), total lung/pleura/ches wall procedures (CHEST), esophageal resections (ESOPH), video-assisted thoracoscopic surgical procedures (VATS), total general thoracic cases 


\section{Abbreviations and Acronyms \\ ABTS = American Board of Thoracic Surgery \\ ACGME $=$ American Council on Graduate Medical Education \\ ANOVA $=$ analysis of variance \\ NRMP = National Resident Matching Program \\ RRC-TS $=$ Residency Review Committee for Thoracic Surgery}

(THOR), congenital (CONG), acquired valvular (VALVE), myocardial revascularization $(\mathrm{CABG})$, reoperations (REDO), and total cardiothoracic cases (TOTAL).

\section{Resident Programs}

Residency programs were categorized by size (number of residents per postgraduate year) and duration (number of years of training). These 2 classifications were then combined as "number of years and number of residents per year." For example a 2-year program is indicated by $2 \mathrm{Y}$, and 2 residents per year program is indicated by $2 \mathrm{R}$. A 2-year program having 2 residents per year would be $2 \mathrm{Y} 2 \mathrm{R}$. Operative experience was then evaluated for each of the 6 models of current cardiothoracic education in the United States: 2Y1R, 2Y2R, 2Y3R, 3Y1R, 3Y2R, and 3Y3R.

\section{Statistical Analysis}

The data were combined in a database and statistically analyzed when appropriate, and are expressed as mean \pm standard deviation, count, or percentage where applicable. Statistical analysis was performed using SAS v. 9.1.3 (SAS Inc, Cary, NC). Student $t$ test was used to compare 2 groups. Analysis of variance (ANOVA) and repeated-measures ANOVA were used for comparison of 3 groups where applicable. Tukey's multiple comparison test was used to identify differences within 3 groups. ABTS examinations were evaluated by Fisher's $t$ test and chi-square test where applicable. Statistical analysis was independently performed by a university statistician (co-author H.H.).

\section{RESULTS}

Table 1 shows the NRMP thoracic surgery match data for the residents entering training in the years from 1996 to 2008. Since 1996, the number of US medical school graduate applicants for thoracic surgery positions has decreased from 156 to 67 in 2008. During this same period, the number of training positions decreased only slightly from 146 to 130 positions. From 1996 to 2008, the NRMP match rate for positions has decreased from $95.9 \%$ to $66.9 \%$, and the match rate for programs has decreased from $93.5 \%$ to $60.9 \%$. When we looked at the number of available positions that filled with US medical school graduates, these have decreased from $80.8 \%$ to less than $50 \%$ for the last 3 years.

Just 4 years ago, match year 2004, there were 161 applicants for 141 ACGME-approved positions in the 95 existing residency programs. More than $93 \%$ of the available positions were filled on match day. In 2008, there were 96 applicants for 130 ACGME-approved positions in the 92 existing programs. Less than $67 \%$ of the available positions were filled on match day. Moreover, in 2004, $75.9 \%$ of the available positions were matched with graduates of US medical schools, whereas in 2008 only $47.7 \%$ of the available positions filled with US medical school graduates. The unmatched rate of applicants, which reflects the selectivity from the applicant pool, has also decreased. In 1996, $28.9 \%$ of the applicants did not match categoric ACGME training positions, and, as recently as 2004, the unmatched applicant rate was $18 \%$. During the last 4 years, it has decreased to less than $10 \%$. Stated differently, more than $90 \%$ of those submitting a rank list will match in a cardiothoracic surgery program.

During the period 2002 to 2007, 795 residents were qualified to enter the ABTS certification process. Of these, 627 had finished $2 Y$ programs and 168 had finished 3 Y programs $(P<.01)$. Figure E1 shows the distribution of residents in this study by the duration and size of their program. The most common program was a $2 \mathrm{Y} 2 \mathrm{R}$, followed in decreasing order by 2Y1R, 2Y3R, 3Y1R, 3Y2R, and 3Y3R.

Table 2 shows a comparison of the average number of major thoracic and cardiac procedures performed by thoracic residents finishing 2- and 3-year programs. Repeated-measures ANOVA and a Student $t$ test were used for this analysis. The first $P$ value column $(P l)$ represents whether $2 \mathrm{Y}$ and $3 \mathrm{Y}$ programs changed in volume during the last 6 years. The second $P$ value column $(P 2)$ represents whether the volume of cases in $2 \mathrm{Y}$ and $3 \mathrm{Y}$ programs were different. The third $P$ value column (P3) represents whether $2 \mathrm{Y}$ and $3 \mathrm{Y}$ programs are changing in different directions over the period studied. During the last 6 years, resident operative volume in PULM, CHEST, VATS, THOR, CONG, CABG, and TOTAL categories have changed significantly over time in both $2 \mathrm{Y}$ and $3 \mathrm{Y}$ programs. All 10 ABTS categories by 2007 were statistically different between the 2- and 3-year programs. There was a statistically different rate of change in operative volume during the 6 years between 2- and 3-year programs in PULM, CHEST, VATS, THOR, CONG, and TOTAL categories. $3 \mathrm{Y}$ programs had statistically significant increases in resident operative volumes in the PULM, CHEST, VATS, THOR, and TOTAL categories than 2-year programs. Residents from $3 \mathrm{Y}$ programs had a higher total number of CONG and CABG cases, but both $3 \mathrm{Y}$ and $2 \mathrm{Y}$ programs had a significant decrease in operative volumes in these categories. The decrease in CONG operations in $3 \mathrm{Y}$ programs was significantly different from that of $2 \mathrm{Y}$ programs.

Table 3 shows a comparison of the average number of major thoracic and cardiac procedures performed by residents finishing from $1 \mathrm{R}, 2 \mathrm{R}$, and $3 \mathrm{R}$ programs. Repeated-measures ANOVA and Tukey's multiple comparison test were performed to evaluate for differences between the 3 sizes of programs. The first $P$ value $(P l)$ column compares the operative volumes in the 3 sizes of programs for differences over the last 6 years. The second $P$ value $(P 2)$ column represents whether the 3 sizes of programs are changing in 
TABLE 1. National Resident Matching Program thoracic surgery match data from 1996 to 2008

\begin{tabular}{|c|c|c|c|c|c|c|c|c|c|c|c|c|c|}
\hline & 1996 & 1997 & 1998 & 1999 & 2000 & 2001 & 2002 & 2003 & 2004 & 2005 & 2006 & 2007 & 2008 \\
\hline Certified program & 93 & 92 & 94 & 90 & 92 & 91 & 95 & 94 & 95 & 93 & 95 & 92 & 92 \\
\hline Programs unfilled & 6 & 11 & 5 & 8 & 10 & 5 & 11 & 15 & 7 & 17 & 31 & 34 & 36 \\
\hline Certified positions & 146 & 143 & 138 & 137 & 139 & 141 & 144 & 144 & 141 & 138 & 139 & 126 & 130 \\
\hline Positions unfilled & 6 & 11 & 5 & 9 & 11 & 6 & 13 & 21 & 9 & 17 & 39 & 42 & 43 \\
\hline Certified applicant & 197 & 176 & 175 & 156 & 156 & 148 & 149 & 145 & 161 & 134 & 104 & 91 & 96 \\
\hline Matched applicants & 140 & 132 & 133 & 128 & 128 & 135 & 131 & 123 & 132 & 121 & 100 & 84 & 87 \\
\hline Unmatched applicants & 57 & 44 & 42 & 28 & 28 & 13 & 18 & 22 & 29 & 13 & 4 & 7 & 9 \\
\hline US grad certified ROL & 156 & 130 & 123 & 118 & 116 & 114 & 112 & 107 & 124 & 99 & 73 & 66 & 67 \\
\hline US grad matched & 118 & 110 & 107 & 100 & 96 & 104 & 102 & 94 & 107 & 92 & 69 & 60 & 62 \\
\hline US grad unmatched & 38 & 20 & 16 & 18 & 20 & 10 & 10 & 13 & 17 & 7 & 4 & 6 & 5 \\
\hline Programs filled (\%) & 93.5 & 88.0 & 94.7 & 91.1 & 89.1 & 94.5 & 88.4 & 84.0 & 92.6 & 81.7 & 67.4 & 63.0 & 60.9 \\
\hline Positions filled (\%) & 95.9 & 92.3 & 96.4 & 93.4 & 92.1 & 95.7 & 91.0 & 85.4 & 93.6 & 87.7 & 71.9 & 66.7 & 66.9 \\
\hline Matched applicants (\%) & 71.1 & 75.0 & 76.0 & 82.1 & 82.1 & 91.2 & 87.9 & 84.8 & 82.0 & 90.3 & 96.2 & 92.3 & 90.6 \\
\hline Unmatched applicants $(\%)$ & 28.9 & 25.0 & 24.0 & 17.9 & 17.9 & 8.8 & 12.1 & 15.2 & 18.0 & 9.7 & 3.8 & 7.7 & 9.4 \\
\hline $\begin{array}{l}\text { Certified positions filled with } \\
\text { US grads }(\%)\end{array}$ & 80.8 & 76.9 & 77.5 & 73.0 & 69.1 & 73.8 & 70.8 & 65.3 & 75.9 & 66.7 & 49.6 & 47.6 & 47.7 \\
\hline
\end{tabular}

$R O L$, Rank order list.

different ways over time. When comparing resident operative volume by size of programs over the 6-year period, we found a statistically significant difference in the following categories: ESOPH, THOR, VALVE, CABG, and REDO in all sizes of programs. In $2007,2 \mathrm{R}$ programs had the lowest number of THOR cases $(P<$ $.01)$, 1R programs had the lowest number of VALVE and REDO cases $(P<.01)$, and the remaining 7 categories had no statistical difference in operative volume among the 3 sizes of programs.
Tables 4 and 5 show the average number of major procedures among all 6 models of thoracic surgery residency. Repeated-measures ANOVA and Tukey's multiple comparison test were used to identify differences in operative volume between the groups. The first $P$ value column $(P l)$ represents whether the resident groups of the respective year program (ie, 2 or 3 year) were different over the 6-year period. The second $P$ value column $(P 2)$ represents whether the resident case volume of the respective year program is changing in different ways over time. One

TABLE 2. Average number of procedures performed by thoracic surgery residents finishing 2- and 3-year programs from 2002 to 2007

\begin{tabular}{|c|c|c|c|c|c|c|c|c|c|}
\hline & 2002 & 2003 & 2004 & 2005 & 2006 & 2007 & $P 1$ value & $P 2$ value & $P 3$ value \\
\hline 2Y PULM & $67 \pm 31$ & $77 \pm 33$ & $78 \pm 29$ & $73 \pm 27$ & $78 \pm 30$ & $78 \pm 30$ & $<.001$ & $<.001$ & $<.001$ \\
\hline 3Y PULM & $48 \pm 14$ & $101 \pm 47$ & $95 \pm 34$ & $124 \pm 72$ & $117 \pm 36$ & $113 \pm 42$ & & & \\
\hline 2Y CHEST & $116 \pm 24$ & $120 \pm 45$ & $121 \pm 38$ & $115 \pm 38$ & $121 \pm 43$ & $124 \pm 45$ & .035 & $<.001$ & .026 \\
\hline 3Y CHEST & $141 \pm 48$ & $150 \pm 56$ & $145 \pm 46$ & $179 \pm 93$ & $168 \pm 52$ & $172 \pm 62$ & & & \\
\hline 2Y ESOPH & $13 \pm 9$ & $14 \pm 10$ & $12 \pm 10$ & $13 \pm 12$ & $13 \pm 9$ & $15 \pm 12$ & NS & $<.001$ & NS \\
\hline $3 \mathrm{Y}$ ESOPH & $19 \pm 3$ & $20 \pm 11$ & $20 \pm 14$ & $24 \pm 20$ & $22 \pm 12$ & $25 \pm 9$ & & & \\
\hline 2Y VATS & $27 \pm 14$ & $28 \pm 17$ & $30 \pm 19$ & $29 \pm 18$ & $31 \pm 16$ & $35 \pm 18$ & .002 & $<.001$ & .044 \\
\hline 3Y VATS & $11 \pm 1$ & $38 \pm 22$ & $45 \pm 31$ & $56 \pm 38$ & $53 \pm 39$ & $57 \pm 42$ & & & \\
\hline 2Y THOR & $160 \pm 52$ & $166 \pm 68$ & $168 \pm 68$ & $162 \pm 57$ & $167 \pm 62$ & $175 \pm 67$ & .012 & $<.001$ & .019 \\
\hline 3Y THOR & $201 \pm 75$ & $212 \pm 71$ & $207 \pm 66$ & $255 \pm 117$ & $236 \pm 76$ & $245 \pm 85$ & & & \\
\hline $2 \mathrm{Y} \mathrm{CONG}$ & $25 \pm 13$ & $23 \pm 13$ & $23 \pm 13$ & $21 \pm 10$ & $22 \pm 12$ & $20 \pm 10$ & $<.001$ & $<.001$ & .003 \\
\hline $3 \mathrm{Y}$ CONG & $64 \pm 26$ & $43 \pm 21$ & $32 \pm 25$ & $32 \pm 34$ & $30 \pm 17$ & $32 \pm 28$ & & & \\
\hline 2Y VALVE & $51 \pm 31$ & $50 \pm 29$ & $55 \pm 38$ & $57 \pm 35$ & $52 \pm 33$ & $55 \pm 34$ & NS & $<.001$ & NS \\
\hline 3Y VALVE & $82 \pm 43$ & $72 \pm 34$ & $82 \pm 40$ & $78 \pm 34$ & $83 \pm 39$ & $65 \pm 24$ & & & \\
\hline 2Y CABG & $135 \pm 56$ & $130 \pm 48$ & $106 \pm 39$ & $111 \pm 47$ & $102 \pm 44$ & $94 \pm 38$ & $<.001$ & $<.001$ & .078 \\
\hline $3 Y \mathrm{CABG}$ & $199 \pm 104$ & $161 \pm 56$ & $182 \pm 88$ & $166 \pm 77$ & $151 \pm 62$ & $128 \pm 49$ & & & \\
\hline 2Y REDO & $12 \pm 7$ & $11 \pm 7$ & $11 \pm 7$ & $12 \pm 9$ & $11 \pm 8$ & $11 \pm 9$ & NS & $<.001$ & NS \\
\hline 3Y REDO & $10 \pm 1$ & $16 \pm 11$ & $19 \pm 15$ & $18 \pm 8$ & $18 \pm 15$ & $15 \pm 9$ & & & \\
\hline 2Y TOTAL & $522 \pm 148$ & $563 \pm 123$ & $542 \pm 126$ & $530 \pm 123$ & $529 \pm 111$ & $532 \pm 119$ & $<.001$ & $<.001$ & .004 \\
\hline 3Y TOTAL & $593 \pm 144$ & $714 \pm 159$ & $743 \pm 131$ & $798 \pm 213$ & $752 \pm 108$ & $720 \pm 178$ & & & \\
\hline
\end{tabular}

NS, Not significant. $P 1=$ difference in volume over time. $P 2=$ difference between $2 \mathrm{Y}$ and $3 \mathrm{Y}$ ABTS category volume. $P 3=$ difference in rate of volume change between $2 \mathrm{Y}$ and $3 \mathrm{Y}$ programs. 
TABLE 3. Average number of procedures performed by thoracic residents finishing 1 resident, 2 residents, and 3 residents per year programs

\begin{tabular}{|c|c|c|c|c|c|c|c|c|}
\hline & 2002 & 2003 & 2004 & 2005 & 2006 & 2007 & P1 value & P2 value \\
\hline 1R PULM & $58 \pm 26$ & $80 \pm 37$ & $81 \pm 28$ & $100 \pm 64$ & $84 \pm 29$ & $88 \pm 35$ & NS & NS \\
\hline 2R PULM & $64 \pm 31$ & $81 \pm 36$ & $80 \pm 27$ & $77 \pm 33$ & $84 \pm 33$ & $83 \pm 32$ & & \\
\hline 3R PULM & $68 \pm 29$ & $87 \pm 40$ & $85 \pm 40$ & $80 \pm 38$ & $84 \pm 42$ & $86 \pm 41$ & & \\
\hline 1R CHEST & $121 \pm 37$ & $120 \pm 44$ & $122 \pm 35$ & $148 \pm 83$ & $125 \pm 37$ & $137 \pm 49$ & NS & NS \\
\hline 2R CHEST & $121 \pm 38$ & $122 \pm 44$ & $124 \pm 33$ & $119 \pm 43$ & $126 \pm 42$ & $128 \pm 46$ & & \\
\hline 3R CHEST & $125 \pm 44$ & $138 \pm 59$ & $137 \pm 56$ & $128 \pm 55$ & $135 \pm 65$ & $138 \pm 63$ & & \\
\hline 1R ESOPH & $13 \pm 6$ & $12 \pm 8$ & $10 \pm 6$ & $13 \pm 10$ & $12 \pm 7$ & $13 \pm 8$ & $<.001$ & NS \\
\hline 2R ESOPH & $10 \pm 7$ & $13 \pm 9$ & $14 \pm 12$ & $15 \pm 16$ & $14 \pm 11$ & $18 \pm 16$ & & \\
\hline 3R ESOPH & $18 \pm 12$ & $20 \pm 15$ & $19 \pm 15$ & $20 \pm 16$ & $18 \pm 13$ & $21 \pm 16$ & & \\
\hline 1R VATS & $24 \pm 13$ & $29 \pm 17$ & $37 \pm 25$ & $45 \pm 37$ & $33 \pm 18$ & $43 \pm 29$ & NS & NS \\
\hline 2R VATS & $29 \pm 16$ & $31 \pm 18$ & $30 \pm 16$ & $30 \pm 17$ & $34 \pm 16$ & $36 \pm 16$ & & \\
\hline 3R VATS & $26 \pm 14$ & $30 \pm 21$ & $34 \pm 28$ & $33 \pm 23$ & $37 \pm 34$ & $40 \pm 31$ & & \\
\hline 1R THOR & $166 \pm 51$ & $169 \pm 59$ & $169 \pm 48$ & $207 \pm 104$ & $174 \pm 53$ & $192 \pm 70$ & .044 & NS \\
\hline 2R THOR & $166 \pm 65$ & $167 \pm 61$ & $173 \pm 51$ & $168 \pm 68$ & $173 \pm 61$ & $179 \pm 70$ & & \\
\hline 3R THOR & $179 \pm 65$ & $197 \pm 91$ & $195 \pm 83$ & $185 \pm 80$ & $192 \pm 94$ & $201 \pm 88$ & & \\
\hline 1R CONG & $26 \pm 19$ & $32 \pm 23$ & $24 \pm 17$ & $25 \pm 29$ & $24 \pm 16$ & $24 \pm 14$ & NS & NS \\
\hline 2R CONG & $28 \pm 13$ & $27 \pm 14$ & $25 \pm 13$ & $24 \pm 13$ & $25 \pm 14$ & $21 \pm 10$ & & \\
\hline 3R CONG & $23 \pm 10$ & $21 \pm 9$ & $25 \pm 21$ & $21 \pm 13$ & $19 \pm 7$ & $22 \pm 24$ & & \\
\hline 1R VALVE & $51 \pm 32$ & $50 \pm 22$ & $52 \pm 36$ & $53 \pm 28$ & $50 \pm 28$ & $46 \pm 21$ & $<.001$ & NS \\
\hline 2R VALVE & $60 \pm 36$ & $57 \pm 32$ & $60 \pm 35$ & $61 \pm 37$ & $58 \pm 37$ & $60 \pm 31$ & & \\
\hline 3R VALVE & $65 \pm 42$ & $57 \pm 39$ & $77 \pm 50$ & $73 \pm 40$ & $66 \pm 40$ & $66 \pm 42$ & & \\
\hline 1R CABG & $158 \pm 90$ & $152 \pm 54$ & $134 \pm 81$ & $135 \pm 58$ & $119 \pm 59$ & $103 \pm 40$ & .013 & NS \\
\hline 2R CABG & $148 \pm 64$ & $135 \pm 50$ & $119 \pm 47$ & $116 \pm 46$ & $99 \pm 35$ & $101 \pm 36$ & & \\
\hline 3R CABG & $142 \pm 63$ & $118 \pm 43$ & $114 \pm 52$ & $125 \pm 79$ & $112 \pm 53$ & $100 \pm 53$ & & \\
\hline 1R REDO & $10 \pm 5$ & $12 \pm 7$ & $11 \pm 7$ & $11 \pm 6$ & $11 \pm 10$ & $10 \pm 6$ & .007 & NS \\
\hline 2R REDO & $11 \pm 7$ & $13 \pm 10$ & $14 \pm 12$ & $14 \pm 9$ & $11 \pm 9$ & $13 \pm 11$ & & \\
\hline 3R REDO & $14 \pm 7$ & $12 \pm 7$ & $16 \pm 9$ & $17 \pm 12$ & $15 \pm 10$ & $13 \pm 9$ & & \\
\hline 1R TOTAL & $521 \pm 154$ & $586 \pm 145$ & $570 \pm 163$ & $620 \pm 209$ & $554 \pm 135$ & $569 \pm 167$ & NS & NS \\
\hline 2R TOTAL & $535 \pm 148$ & $596 \pm 166$ & $578 \pm 578$ & $561 \pm 176$ & $555 \pm 147$ & $563 \pm 149$ & & \\
\hline 3R TOTAL & $568 \pm 146$ & $600 \pm 107$ & $628 \pm 135$ & $613 \pm 173$ & $595 \pm 126$ & $587 \pm 146$ & & \\
\hline
\end{tabular}

$N S$, Not significant. $P 1=$ difference in volume between the 3 programs over time. $P 2=$ difference in the rate of change in volume between the 3 programs. ANOVA.

limitation of this analysis is that the sample size in 3-year programs might have been too small to define all differences. Therefore, we may be missing differences among 3-year programs that would be present if the sample size was larger. $2 \mathrm{Y} 2 \mathrm{R}$ programs had the lowest average number of cases in 5 of the 10 categories, reaching statistical significance in CHEST, THOR, and TOTAL. 2Y1R programs had the lowest average operative volume in 4 of the 10 categories, with the volumes reaching statistical significance in ESOPH, VALVE, and REDO. 3Y3R programs had the highest average operative volume in 6 of the 10 categories. 3Y1R programs had the highest number of CONG cases but had significantly less ESOPH volume compared with their respective year programs (ie, 3Y2R, 3Y3R) $(P<.01)$. 3Y2R programs had the highest average resident volume in 3 of the 10 categories, but they had significantly less VATS and CABG volume than their $3 \mathrm{Y}$ peers $(P<.01)$.

Of the 795 residents who qualified for the written ABTS examination, $793(99 \%)$ took the examination and 2 did not show up for the examination. The passing rate for all 793 candidates during the 6 -year period was $87.8 \%$. The passing rate was higher in residents from 3-year programs throughout the period of the study, but comparing the groups within each year, the difference reached statistical significance only in $2006(80 \%$ vs $100 \%$, respectively, $P=.014)$. During the 6 -year period, there was a significant difference in the passing rate of the written examination when stratified by 2 - and 3 -year programs $(85.9 \%$ vs $94.6 \%$, respectively, $P=.003)$ (Table 6).

When looking at the ATBS examinations for all takers in 2003 , approximately $90 \%$ of the candidates taking the ABTS written examination passed. In 2007, less than $84 \%$ passed. In 2003, $85 \%$ of those taking the oral examination passed, whereas in 2007 only $71 \%$ passed. Compared with the oral examination pass rate in 2006 , there was a statistically significant decrease in 2007 (86\% vs $71 \%$, respectively; $P<.01)$. By looking at the trend over time, there is a steady decline in the passing rate on both the written and the oral boards, but this does not reach statistical significance over the period of the study.

\section{DISCUSSION}

The practice of cardiothoracic surgery has changed significantly over the past decade. Although many factors have 
TABLE 4. Average number of thoracic procedures among the 6 current thoracic education programs

\begin{tabular}{|c|c|c|c|c|c|c|c|c|}
\hline & 2002 & 2003 & 2004 & 2005 & 2006 & 2007 & $P 1$ value & $P 2$ value \\
\hline \multicolumn{9}{|c|}{ Pulmonary resections (PULM) } \\
\hline $2 \mathrm{y} 1 \mathrm{r}$ & $63 \pm 29$ & $73 \pm 25$ & $80 \pm 25$ & $83 \pm 26$ & $82 \pm 29$ & $84 \pm 31$ & NS & NS \\
\hline $2 \mathrm{y} 2 \mathrm{r}$ & $70 \pm 32$ & $74 \pm 29$ & $75 \pm 26$ & $67 \pm 24$ & $74 \pm 26$ & $70 \pm 22$ & & \\
\hline $2 y 3 r$ & $69 \pm 31$ & $86 \pm 42$ & $79 \pm 37$ & $72 \pm 32$ & $76 \pm 36$ & $82 \pm 36$ & & \\
\hline $3 y 1 \mathrm{r}$ & $49 \pm 15$ & $100 \pm 56$ & $84 \pm 35$ & $146 \pm 103$ & $96 \pm 26$ & $100 \pm 45$ & NS & NS \\
\hline $3 y 2 r$ & $42 \pm 14$ & $105 \pm 47$ & $96 \pm 24$ & $113 \pm 40$ & $119 \pm 36$ & $127 \pm 24$ & & \\
\hline $3 y 3 r$ & $59 \pm 7$ & $95 \pm 24$ & $115 \pm 49$ & $107 \pm 45$ & $156 \pm 16$ & $116 \pm 60$ & & \\
\hline \multicolumn{9}{|c|}{ Lung/pleura/chest (CHEST) } \\
\hline $2 \mathrm{y} 1 \mathrm{r}$ & $111 \pm 26$ & $110 \pm 28$ & $121 \pm 34$ & $123 \pm 31$ & $123 \pm 37$ & $129 \pm 44$ & .01 & NS \\
\hline $2 \mathrm{y} 2 \mathrm{r}$ & $117 \pm 34$ & $114 \pm 37$ & $115 \pm 31$ & $107 \pm 32$ & $115 \pm 34$ & $112 \pm 32$ & & \\
\hline $2 \mathrm{y} 3 \mathrm{r}$ & $120 \pm 41$ & $136 \pm 61$ & $129 \pm 48$ & $120 \pm 51$ & $124 \pm 57$ & $131 \pm 55$ & & \\
\hline $3 y 1 \mathrm{r}$ & $142 \pm 49$ & $149 \pm 64$ & $127 \pm 39$ & $211 \pm 132$ & $139 \pm 35$ & $157 \pm 59$ & NS & NS \\
\hline $3 y 2 r$ & $134 \pm 50$ & $151 \pm 57$ & $147 \pm 30$ & $163 \pm 52$ & $168 \pm 47$ & $184 \pm 46$ & & \\
\hline $3 y 3 r$ & $154 \pm 52$ & $150 \pm 42$ & $174 \pm 79$ & $155 \pm 62$ & $237 \pm 38$ & $186 \pm 94$ & & \\
\hline \multicolumn{9}{|c|}{ Esophageal operations (ESOPH) } \\
\hline $2 \mathrm{y} 1 \mathrm{r}$ & $13 \pm 6$ & $11 \pm 8$ & $10 \pm 7$ & $11 \pm 8$ & $11 \pm 7$ & $12 \pm 7$ & $<.001$ & NS \\
\hline $2 \mathrm{y} 2 \mathrm{r}$ & $10 \pm 7$ & $11 \pm 7$ & $11 \pm 8$ & $11 \pm 9$ & $12 \pm 8$ & $14 \pm 10$ & & \\
\hline $2 \mathrm{y} 3 \mathrm{r}$ & $18 \pm 12$ & $19 \pm 14$ & $17 \pm 15$ & $19 \pm 17$ & $16 \pm 12$ & $20 \pm 16$ & & \\
\hline $3 y 1 \mathrm{r}$ & $19 \pm 3$ & $15 \pm 8$ & $12 \pm 5$ & $20 \pm 13$ & $14 \pm 7$ & $17 \pm 9$ & .001 & NS \\
\hline $3 y 2 r$ & & $21 \pm 12$ & $24 \pm 16$ & $30 \pm 28$ & $24 \pm 13$ & $32 \pm 26$ & & \\
\hline $3 y 3 r$ & & $29 \pm 15$ & $25 \pm 15$ & $24 \pm 15$ & $33 \pm 8$ & $30 \pm 16$ & & \\
\hline \multicolumn{9}{|l|}{ VATS } \\
\hline $2 \mathrm{y} 1 \mathrm{r}$ & $25 \pm 13$ & $26 \pm 13$ & $32 \pm 21$ & $32 \pm 20$ & $32 \pm 15$ & $37 \pm 17$ & NS & NS \\
\hline $2 \mathrm{y} 2 \mathrm{r}$ & $29 \pm 16$ & $30 \pm 17$ & $30 \pm 17$ & $29 \pm 17$ & $32 \pm 14$ & $32 \pm 14$ & & \\
\hline $2 \mathrm{y} 3 \mathrm{r}$ & $26 \pm 14$ & $28 \pm 20$ & $29 \pm 19$ & $27 \pm 19$ & $28 \pm 18$ & $36 \pm 21$ & & \\
\hline $3 y 1 \mathrm{r}$ & $11 \pm 1$ & $38 \pm 24$ & $52 \pm 34$ & $77 \pm 50$ & $41 \pm 29$ & $58 \pm 47$ & .004 & NS \\
\hline $3 y 2 r$ & & $35 \pm 22$ & $32 \pm 13$ & $37 \pm 16$ & $40 \pm 21$ & $49 \pm 16$ & & \\
\hline $3 y 3 r$ & & $46 \pm 21$ & $61 \pm 49$ & $53 \pm 27$ & $115 \pm 46$ & $68 \pm 66$ & & \\
\hline \multicolumn{9}{|c|}{ Total thoracic (THOR) } \\
\hline $2 \mathrm{y} 1 \mathrm{r}$ & $154 \pm 41$ & $155 \pm 44$ & $165 \pm 47$ & $172 \pm 47$ & $169 \pm 53$ & $181 \pm 64$ & $<.001$ & NS \\
\hline $2 \mathrm{y} 2 \mathrm{r}$ & $156 \pm 54$ & $156 \pm 55$ & $159 \pm 46$ & $150 \pm 50$ & $158 \pm 49$ & $156 \pm 50$ & & \\
\hline $2 y 3 r$ & $171 \pm 60$ & $193 \pm 93$ & $183 \pm 74$ & $173 \pm 74$ & $176 \pm 84$ & $190 \pm 81$ & & \\
\hline $3 \mathrm{y} 1 \mathrm{r}$ & $192 \pm 62$ & $209 \pm 76$ & $180 \pm 51$ & $296 \pm 151$ & $198 \pm 50$ & $222 \pm 80$ & NS & NS \\
\hline $3 y 2 r$ & $201 \pm 90$ & $207 \pm 68$ & $210 \pm 234$ & $234 \pm 231$ & $231 \pm 72$ & $261 \pm 75$ & & \\
\hline $3 y 3 r$ & $233 \pm 81$ & $231 \pm 80$ & $254 \pm 110$ & $225 \pm 92$ & $337 \pm 48$ & $270 \pm 117$ & & \\
\hline
\end{tabular}

$N S$, Not significant. $P 1=$ difference in operative volume over time. $P 2=$ difference in the rate of change in operative volume between programs. ANOVA.

influenced that change, the emergence of less-invasive technologies have dramatically altered the caseload, such as coated stents, atrial septal defect closure devices, endovascular grafts, advanced computed tomography/magnetic resonance imaging, endoscopy, radiation therapy, and interventional bronchoscopy. These new less-invasive technologies have allowed cardiologists to intervene in coronary artery disease and now valvular disease. Radiologists and pulmonologists can now treat pleural space diseases with computed tomography and ultrasound guidance. Vascular surgeons now treat diseases of the thoracic aorta and arch. Radiation oncologists can treat lung cancer with radiofrequency ablation. Cardiothoracic diseases still exist, but less-invasive technologies have allowed other specialties to treat them. ${ }^{6}$ In addition, a study of this type was not needed to tell us that cardiothoracic surgery has lost much of the appeal it once had as a career choice. If the pass/fail rates on the ABTS examinations were any indication, not only is the specialty attracting fewer applicants, those who do apply as a group are less capable.

The educational/training process of a cardiothoracic surgeon might be compared with the tailoring of a fine suit. First, the tailor must find and acquire the finest in fabric, weave, and color (the applicant). In this context, the importance of applicants cannot be understated because they are the "life blood of the specialty.", Next, the master tailor and his/her staff (program director and faculty) must put in time, effort, and passion to make the best suit possible. Finally, the store owner (RRC-TS, ABTS examination process) must examine the suit and determine that it is indeed the best suit in the world, and then he/she can proudly display it in his/her store for the world to see and buy. ${ }^{8}$ Although this is a simplification of the educational process, it highlights the importance of each part of the process. Our conclusions and recommendation follow this model. 
TABLE 5. Comparison of the average number of cardiac procedures among the 6 current thoracic education models

\begin{tabular}{|c|c|c|c|c|c|c|c|c|}
\hline & 2002 & 2003 & 2004 & 2005 & 2006 & 2007 & $P 1$ value & $P 2$ value \\
\hline \multicolumn{9}{|c|}{ Congenital (CONG) } \\
\hline $2 \mathrm{y} 1 \mathrm{r}$ & $23 \pm 15$ & $25 \pm 17$ & $21 \pm 14$ & $19 \pm 10$ & $21 \pm 18$ & $21 \pm 14$ & NS & NS \\
\hline $2 y 2 r$ & $28 \pm 13$ & $23 \pm 13$ & $25 \pm 14$ & $23 \pm 11$ & $26 \pm 15$ & $20 \pm 10$ & & \\
\hline $2 \mathrm{y} 3 \mathrm{r}$ & $23 \pm 10$ & $19 \pm 8$ & $22 \pm 9$ & $19 \pm 8$ & $19 \pm 7$ & $17 \pm 6$ & & \\
\hline $3 y 1 r$ & $64 \pm 26$ & $51 \pm 51$ & $34 \pm 34$ & $41 \pm 51$ & $45 \pm 18$ & $29 \pm 14$ & NS & NS \\
\hline $3 y 2 r$ & & $39 \pm 14$ & $26 \pm 10$ & $26 \pm 18$ & $23 \pm 10$ & $24 \pm 10$ & & \\
\hline $3 y 3 r$ & & $31 \pm 12$ & $42 \pm 48$ & $29 \pm 22$ & $18 \pm 10$ & $54 \pm 60$ & & \\
\hline \multicolumn{9}{|c|}{ Acquired valvular (VALVE) } \\
\hline $2 \mathrm{y} 1 \mathrm{r}$ & $39 \pm 21$ & $46 \pm 19$ & $41 \pm 22$ & $47 \pm 26$ & $43 \pm 20$ & $40 \pm 16$ & $<.001$ & NS \\
\hline $2 \mathrm{y} 2 \mathrm{r}$ & $49 \pm 22$ & $46 \pm 23$ & $48 \pm 27$ & $52 \pm 32$ & $49 \pm 31$ & $58 \pm 33$ & & \\
\hline $2 \mathrm{y} 3 \mathrm{r}$ & $65 \pm 44$ & $60 \pm 41$ & $83 \pm 52$ & $75 \pm 43$ & $69 \pm 41$ & $65 \pm 44$ & & \\
\hline $3 y 1 r$ & $76 \pm 39$ & $59 \pm 27$ & $88 \pm 48$ & $71 \pm 28$ & $85 \pm 41$ & $62 \pm 24$ & .03 & NS \\
\hline $3 y 2 r$ & $101 \pm 48$ & $98 \pm 27$ & $92 \pm 35$ & $94 \pm 38$ & $95 \pm 36$ & $66 \pm 22$ & & \\
\hline $3 y 3 r$ & $62 \pm 36$ & $37 \pm 9$ & $50 \pm 16$ & $64 \pm 31$ & $47 \pm 27$ & $73 \pm 27$ & & \\
\hline \multicolumn{9}{|c|}{ Myocardial revascularization (CABG) } \\
\hline $2 \mathrm{y} 1 \mathrm{r}$ & $124 \pm 46$ & $143 \pm 52$ & $104 \pm 35$ & $123 \pm 49$ & $106 \pm 51$ & $91 \pm 30$ & NS & NS \\
\hline $2 \mathrm{y} 2 \mathrm{r}$ & $136 \pm 56$ & $127 \pm 46$ & $107 \pm 40$ & $110 \pm 44$ & $94 \pm 34$ & $99 \pm 35$ & & \\
\hline $2 y 3 r$ & $146 \pm 64$ & $119 \pm 43$ & $107 \pm 45$ & $101 \pm 48$ & $105 \pm 45$ & $92 \pm 47$ & & \\
\hline $3 \mathrm{y} 1 \mathrm{r}$ & $232 \pm 117$ & $176 \pm 52$ & $234 \pm 110$ & $164 \pm 69$ & $182 \pm 61$ & $134 \pm 46$ & .021 & NS \\
\hline $3 y 2 r$ & $188 \pm 78$ & $164 \pm 57$ & $152 \pm 49$ & $143 \pm 49$ & $118 \pm 34$ & $109 \pm 39$ & & \\
\hline $3 y 3 r$ & $114 \pm 55$ & $108 \pm 47$ & $148 \pm 79$ & $205 \pm 112$ & $169 \pm 91$ & $150 \pm 69$ & & \\
\hline \multicolumn{9}{|c|}{ Reoperations (REDO) } \\
\hline $2 \mathrm{y} 1 \mathrm{r}$ & $10 \pm 5$ & $12 \pm 8$ & $9 \pm 5$ & $9 \pm 4$ & $9 \pm 6$ & $9 \pm 4$ & $<.001$ & NS \\
\hline $2 \mathrm{y} 2 \mathrm{r}$ & $11 \pm 7$ & $10 \pm 7$ & $10 \pm 4$ & $11 \pm 8$ & $9 \pm 6$ & $13 \pm 11$ & & \\
\hline $2 \mathrm{y} 3 \mathrm{r}$ & $14 \pm 7$ & $12 \pm 8$ & $17 \pm 9$ & $18 \pm 13$ & $15 \pm 10$ & $13 \pm 10$ & & \\
\hline $3 y 1 \mathrm{r}$ & $10 \pm 1$ & $11 \pm 5$ & $18 \pm 8$ & $15 \pm 8$ & $21 \pm 19$ & $13 \pm 9$ & NS & NS \\
\hline $3 y 2 r$ & & $24 \pm 12$ & $24 \pm 19$ & $23 \pm 8$ & $18 \pm 14$ & $16 \pm 9$ & & \\
\hline $3 y 3 r$ & & $8 \pm 2$ & $9 \pm 5$ & $15 \pm 6$ & $12 \pm 7$ & $15 \pm 8$ & & \\
\hline \multicolumn{9}{|c|}{ Total cardiothoracic (TOTAL) } \\
\hline $2 \mathrm{y} 1 \mathrm{r}$ & $477 \pm 135$ & $541 \pm 102$ & $510 \pm 100$ & $527 \pm 98$ & $518 \pm 106$ & $521 \pm 123$ & $<.001$ & NS \\
\hline $2 \mathrm{y} 2 \mathrm{r}$ & $513 \pm 146$ & $550 \pm 141$ & $522 \pm 140$ & $505 \pm 134$ & $509 \pm 128$ & $516 \pm 109$ & & \\
\hline $2 \mathrm{y} 3 \mathrm{r}$ & $583 \pm 149$ & $600 \pm 110$ & $607 \pm 118$ & $576 \pm 122$ & $567 \pm 96$ & $562 \pm 124$ & & \\
\hline $3 y 1 r$ & $616 \pm 154$ & $707 \pm 177$ & $767 \pm 178$ & $859 \pm 232$ & $735 \pm 123$ & $697 \pm 206$ & NS & NS \\
\hline $3 y 2 r$ & $614 \pm 132$ & $763 \pm 148$ & $727 \pm 51$ & $775 \pm 155$ & $733 \pm 93$ & $733 \pm 151$ & & \\
\hline $3 y 3 r$ & $472 \pm 80$ & $600 \pm 87$ & $735 \pm 176$ & $740 \pm 257$ & $843 \pm 90$ & $749 \pm 190$ & & \\
\hline
\end{tabular}

$N S$, Not significant. $P 1=$ difference in operative volume over time. $P 2=$ difference in the rate of change in operative volume between programs. ANOVA.

\section{The Fabric (Applicants)}

The residency applicant data from the NRMP paints a startling picture. The number of applicants to thoracic surgery residencies in 2008 is approximately half of what it was in
2004, and the numbers were actually decreasing slightly even before then. Are we not hearing what medical students and surgical residents, not to mention the data above, are telling us? We are simply not offering an education that

TABLE 6. Performance of thoracic surgery residents who took the written examination of the American Board of Thoracic Surgery for the first time between 2002 and 2007 and their distribution among 2- and 3-year programs

\begin{tabular}{|c|c|c|c|c|c|c|c|c|c|c|}
\hline \multirow[b]{2}{*}{ Year } & \multicolumn{3}{|c|}{ All candidates } & \multicolumn{3}{|c|}{ 2-y programs } & \multirow[b]{2}{*}{$P$ value } & \multicolumn{3}{|c|}{ 3-y programs } \\
\hline & Total & Pass $(\%)$ & Fail & Total & Pass $(\%)$ & Fail & & Total & Pass $(\%)$ & Fail \\
\hline 2007 & 128 & $112(87.5)$ & 16 & 101 & $87(86.1)$ & 14 & NS & 27 & 25 (93.6) & 2 \\
\hline 2006 & 147 & $123(83.7)$ & 24 & 123 & $99(80.5)$ & 24 & .014 & 24 & $24(100)$ & 0 \\
\hline 2005 & 136 & $119(87.5)$ & 17 & 102 & $88(86.3)$ & 14 & NS & 34 & $31(91.2)$ & 3 \\
\hline 2004 & 117 & 99 (84.6) & 18 & 91 & $75(82.4)$ & 16 & NS & 26 & $24(92.3)$ & 2 \\
\hline 2003 & 127 & $120(94.5)$ & 7 & 101 & $94(93.1)$ & 7 & NS & 26 & $26(100)$ & 0 \\
\hline 2002 & 138 & $123(89.1)$ & 15 & 108 & $95(88.0)$ & 13 & NS & 30 & $28(93.3)$ & 2 \\
\hline $2002-2007$ & 793 & $696(87.8)$ & 97 & 626 & $538(85.9)$ & 88 & .003 & 167 & 158 (94.6) & 9 \\
\hline
\end{tabular}

NS, Not significant. $P$ value $=$ difference in 2 - and 3-year pass rates. 
will adequately prepare surgeons for practice in today's environment. ${ }^{9}$ Whether it is endovascular technologies, robotics, endobronchial and endoesophageal procedures, or something not yet conceived, we, the educators in thoracic surgery, need to dramatically change our methodologies.

Medical students and residents are not choosing to enter the field of cardiothoracic surgery for many reasons, including lifestyle, length of training, perceived shortcomings in training, decreased reimbursement, and loss of prestige of the field. In addition, the job market has significantly worsened. Cardiothoracic diseases are still present in patients, but because we have not trained with less-invasive technologies as our cardiologists, vascular surgeons, radiologists, and pulmonologists have, we have lost the ability to intervene in these patients first. ${ }^{10,11}$ The thoracic surgery residents in this study were in the 1999 to 2005 NRMP match year. The residents matched from 2006 to the present are still in training, so we have yet to see the effect of the continued decline in applicants on case volumes and board pass rates.

Medical specialty training has changed significantly over the past several years, and some medical students now seem to emphasize quality of life issues in their choice of specialty. ${ }^{12}$ Although the 80-hour work week was enacted with patient safety in mind, a by-product of this policy is that residents are no longer permitted to be in the hospital for extended periods of time. The 80-hour work week runs contrary to surgery's history of long hours and dedicated patient follow-up. Cardiothoracic residency programs have struggled to find a balance between resident education and compliance with this mandate. ${ }^{13}$ In this study there was no decrease in resident operative volume that we could attribute to the 80-hour week limit. In fact, 3 Y programs significantly increased and maintained their total operative cases during this period.

\section{The Tailor (Training Programs)}

The residents graduating from $3 \mathrm{Y}$ programs have greater volumes in all case categories when compared with $2 \mathrm{Y}$ programs. This puts aside the notion that a third year is a "waste.' Residents are not left in the laboratory or the intensive care unit, but they are in fact operating and acquiring more knowledge. The most obvious decrease in operative experience in the 2-year training model has been the decrease in myocardial revascularizations. A resident finishing in 2007 would need 3 years to obtain the same myocardial revascularization experience as a resident graduating from a 2 -year program in 2002. This change in training volumes certainly reflects changes in the current practice of cardiothoracic surgery and volumes nationwide. ${ }^{14}$ Surprisingly, there has not been an increase in the number of resident valve cases, as many would have believed. It has also been suggested that academic medical centers, our training centers, are particularly sensitive to this decrease because many revascularizations still occur in hospitals without training obligations. ${ }^{15}$
The ABTS examination pass rates were better for residents from $3 \mathrm{Y}$ programs than $2 \mathrm{Y}$ programs. The data suggest that a $3 \mathrm{Y}$ program would be a preferred model. Indeed, the $2 \mathrm{Y} 2 \mathrm{R}$ programs (the most prevalent size and duration model currently in place in the United States) seems to be the most vulnerable. If the new ABTS requirements that took effect in July of 2007 were applied to the residents who completed their training during the past 6 years, only $15.2 \%$ (36/236) of the 2Y2R residents during the last 6 years had enough case volume in VALVE, CABG, and REDO to qualify using the cardiothoracic track. Likewise, only $10.2 \%$ (24/236) of the 2Y2R residents during the last 6 years would have enough case volume in CHEST and ESOPH to quality for the ABTS examination using the thoracic track. To their credit, residency programs seem to have been able to keep operative volumes at acceptable levels by diversifying the case mix. For example, although the volume of cardiac cases has decreased, the numbers and types of general thoracic cases have increased. What once was a cardiac heavy thoracic residency training has now become more even in operative experience. This has occurred despite residents traditionally still spending more time on the "cardiac" rather than the "thoracic" service. The exception to this is programs that have a defined thoracic track where the resident time changes accordingly. This change in case mix may expand the clinical capabilities of the resident at the end of his/her training. This is an appropriate change because most cardiothoracic surgeons are performing a significant volume of general thoracic surgical procedures in their daily practices. ${ }^{16}$

\section{Store Owners (the Residency Review Committee and American Board of Thoracic Surgery)}

Admittedly, operative case volume is only one measure of the adequacy of a residency program and may not even be the most important one. ${ }^{17-19}$ However, it is a number that can be measured. This study did not correlate case volumes with board scores, but the ABTS policy dictates that candidates who fail to meet the Board's minimum case requirements are not permitted to take the examination. Moreover, the number of index cases performed by residents is closely monitored by the RRC-TS, and consistently low volumes will often precipitate an adverse action from that body. Thus, the numbers are of importance.

Last, the output of the training process is measured by the ABTS. $^{8}$ The criteria for qualifying to sit for the written ABTS examination have changed twice in the last 8 years. ${ }^{20}$ The most recent change increased the numbers and allowed for the creation of defined tracks for cardiothoracic and thoracic training. The ABTS has not tracked applicants' exposure or experience with new technologies. With the exception of VATS, new technologies are not being categorically captured for more than 10 years by the ABTS. Future thoracic residents' evaluation by the ABTS may need to 
include categories for these new technologies that many now believe may be mandatory (valve repair, off pump, endovascular, heart failure). This is particularly relevant because the mixture of cases for graduating residents is not homogenous, and depending on the program that the resident has graduated from, his or her experience and the case mixture can be varied and not reflected in current case indices reporting.

A resident applying for a cardiothoracic residency has the potential to end up in 1 of 36 different types of programs. First, the resident enters 1 of the 6 thoracic residency programs. With the removal of the American Board of Surgery requirement, the resident can be ABS certified or not; this doubles the number of pathways. At the end of the thoracic educational process, the graduating resident can choose to be in a cardiothoracic mix practice or a general thoracic or cardiac only practice. This process allows for too many different suits rather than a few excellent ones, which is what the specialty needs now. With the addition of the 4-year general surgery/3-year cardiothoracic track, as well as the categoric track, it only confuses the matter more for our applicants and field. At a time when the specialty is going through great controversy with applicants and training, we should consolidate the training algorithm, not exponentially expand the number of possible suits for the prospective match candidates: quality, not quantity.

There have been various suggestions over the recent years regarding the state of our educational programs. Most literature on thoracic surgery education is based on editorials, surveys, or anecdotes. ${ }^{21}$ Many authors offer appropriate suggestions and insightful recommendations, but there has been little action and the NRMP results confirm this fact. This study is the first comprehensive review of the thoracic surgery resident case volumes as reported to the ABTS. It demonstrates little homogeneity in the educational process for the 87 residents who are commencing their training in July of 2008, being distributed over 6 resident program models. It is our opinion that thoracic surgery residency programs will be better served by adopting a $3 \mathrm{Y}$ minimum for residencies and by either closing programs that cannot, or do not, provide adequate surgical experience to their residents or reducing their resident complement to allow for more cases per resident. Adopting a "mentor" or preceptor model in which a resident is assigned to an individual surgeon rather than a "service" might encourage greater responsibility for resident education on the part of individual faculty. The concept of a "categoric" thoracic surgery residency, such as that now being evaluated at Stanford, may well be a timely and appropriate consideration.

There is some precedent for a 3-year period of cardiothoracic surgical training, particularly if attention is given to other training programs in the world. France, the United Kingdom, Canada, and Japan all have a minimum of 3 years in cardiothoracic training with an overall similar period of 6 to 7 years of total training. ${ }^{21-25}$ The decision is not so straightforward, however, because required case numbers for residents from these countries are even lower than those of our current graduating residents. ${ }^{23}$ Certainly, it has been suggested that an additional year in cardiothoracic training would allow for adequate training in currently sought after skills that incorporate new technologies, such as endovascular, off-pump, robotics, or valve repair. ${ }^{24}$ Percutaneous and endovascular therapies are improving and progressing beyond coronary disease and involving valvular heart disease. Institutions have to support clinicians in the acquisition of new skills to then impart to trainees. ${ }^{26}$ This would improve a recent graduate's ability to become an immediate asset to his or her new professional group.

How can we prepare ourselves, trainees and surgeons alike, to face the challenges of the future? We can achieve this by a) providing mentorship for medical students and residents early on by demonstrating the excitement and fulfillment of a career in cardiothoracic surgery; b) expanding the cardiothoracic residency experience to a minimum of 3 years; c) reducing resident complement in low-volume programs, so that the number of residents does not exceed the capacity of the program to provide a meaningful experience to each; d) diversifying resident education and program portfolios e) simulating real-life practice by allowing thoracic residents to operate at different sites by shadowing their faculty mentor in an apprenticeship like model; and f) promoting tracking of new technologies in the ABTS operative log.

\section{CONCLUSIONS}

Thoracic surgery residency education is at a critical juncture. The future of our specialty rests in those who are in residencies right now. As teachers and mentors, we have the responsibility of preparing them for the careers that await them. In 2009, it is important not to change thoracic education back to produce the cardiothoracic surgeon of the 1990s, but to produce the best cardiothoracic surgeon for 2010 and beyond. This will take great vision and difficult change, but it is critically important for our survival so that we can best care for our patients and preserve the legacy of our great profession.

\section{References}

1. Kron IL, Mathisen D. Caring for the residents: the view of two program directors. Ann Thorac Surg. 2004;78:17.

2. Pennington DG. The impact of new technology on cardiothoracic surgical practice. Ann Thorac Surg. 2006;81:10-8.

3. Kypson AP, Nifong LW, Chitwood WR. Robot-assisted surgery: training and re-training surgeons. Int J Med Robot. 2004;1:70-6.

4. Grabo DJ, DiMuzio PJ, Kairys JC, et al. Have endovascular procedures negatively impacted general surgery training? Ann Surg. 2007;246:472-80.

5. Vassiliades TA Jr, Block PC, Cohn LH, et al. The clinical development of percutaneous heart valve technology: a position statement of the Society of Thoracic Surgeons (STS), the American Association for Thoracic Surgery (AATS), and the Society of Cardiovascular Angiography and Intervention (SCAI). Catheter Cardiovasc Interv. 2005;65:73-9. 
6. Kouchoukos NT. Cardiothoracic surgery in the new millennium: challenges and opportunities in a time of paradox. Ann Thorac Surg. 2000;69:1303-11.

7. Orringer MB. The Thoracic Surgery Directors Association (TSDA): focus upon curriculum for resident education. Semin Thorac Cardiovasc Surg. 1998;10:173-7.

8. Gay WA Jr. Graduate education in thoracic surgery and the American Board of Thoracic Surgery. Semin Thorac Cardiovasc Surg. 1998;10:178-80.

9. Gardner TJ. Residency training for the future, not the past. Ann Thorac Surg. 2004;78:1519-21.

10. Salazar JD, Lee R, Wheatley GH, et al. Are there enough jobs in cardiothoracic surgery? The Thoracic Surgery Residents Association job placement survey for finishing residents. Ann Thorac Surg. 2004;78:1523-7.

11. Levitsky S. Navigating the new "flat world" of cardiothoracic surgery. Ann Thorac Surg. 2007;83:361-9.

12. Morishita K, Naraoka S, Miyajima M, et al. Medical students' views on thoracic surgery residency programs in a Japanese medical school. Jpn J Thorac Cardiovasc Surg. 2003;51:475-7.

13. Barden CB, Specht MC, McCarter MD, et al. Effects of limited work hours on surgical training. J Am Coll Surg. 2002;195:531-8.

14. STS Executive Summary 2006. Available at: www.sts.org. Accessed January $10,2008$.

15. Verrier ED. Who moved my heart? Adaptive responses to disruptive challenges. J Thorac Cardiovasc Surg. 2004;127:1235-44.

16. Shemin RJ, Dziuban SW, Kaiser LR, et al. Thoracic surgery workforce: snapshot at the end of the twentieth century and implications for the new millennium. Ann Thorac Surg. 2002;73:2014-32.

17. DaRosa DA, Fullerton DA, Kron IL, Orringer MB. Content of thoracic residency training and its relevance to the practice of medicine. Ann Thorac Surg. 2000;69: 1321-6.

18. Grillo HC. Education or training in cardiothoracic surgery? Ann Thorac Surg. 2000;69:1319-20

19. Hance J, Aggarwal R, Stanbridge R, et al. Objective assessment of technical skills in cardiac surgery. Eur J Cardiothorac Surg. 2005;28:157-62.

20. American Board of Thoracic Surgery. Operative case requirements. Available at: www.abts.org/sections/Certification/Operative_Requirements/index.html. Accessed January 2008.

21. Takeuchi K, Otaki M, Kitamura N, et al. Thoracic surgery residency program and health care system in the United States. Jpn J Thorac Cardiovasc Surg. 2004;52: 401-6.

22. O'Kane H. Training of cardiothoracic surgeons in the UK. Jpn J Thorac Cardiovasc Surg. 2005;53:332-5.

23. Acar C. Training in thoracic and cardiovascular surgery in France. Jpn J Thorac Cardiovasc Surg. 2005;53:328-9.

24. Geha AS. Medical education and the training of cardiothoracic surgeons in the United States of America. Jpn J Thorac Cardiovasc Surg. 2004;52:401-6.

25. Chiu RC. Training program for thoracic and cardiovascular surgeons in Canada. Jpn J Thorac Cardiovasc Surg. 2005;53:324-7.

26. Lytle B, Mack M. The future of cardiac surgery. Ann Thorac Surg. 2005;79:1470-2.

\section{Discussion}

Dr Irving Kron (Charlottesville, Va). Dr Prasad, I enjoyed this presentation. I agree entirely that thoracic surgery education is in a crisis. This has been noted very well by you in citing the decrease in applications. Another major concern is the decrease in ABTS pass rates. You did a good job of putting this all together. Unfortunately, that is all I agree with in this article. My differences of opinion relate to several issues. The catchy title speaks to challenges of emerging technologies, yet you don't really get into that at all, in either your methodology or your article. So can you comment on that?

Dr Prasad. The question of emerging technologies probably has to start with Gordon Moore, the cofounder of Intel, who has probably caused a lot of these problems. In 1965 he wrote an article that looked at a retrospective review of transistors, and he made Moore's Law at that point, which said that the amount of transistors per square inch on a circuit board will increase, will double every 2 years, and that was in 1965 . In the last 40 years, that has held up. And what does that mean for us as cardiac surgeons? It means that now it gives the potential for cardiologists to put stents in, to develop stents, and to not involve us in the care of our patients. It allows radiologists to use 64-slice computed tomography scans and aortic stents and not involve us in the care of these patients. It allows interventional pulmonologists to carry portable ultrasounds and drain pleural effusions and not involve us in the care of these patients. And last, it allows medical oncologists to use radiation therapy and not involve us in the care of these patients, and soon they will be treating $\mathrm{T} 1$ lesions with radiofrequency ablation. These emerging technologies have exponentially grown in the last 10 years, and I think the field has not caught up to that. That is the reason we chose the title, and it transcends to volume in practice and obviously transcends to volume in training.

Dr Kron. I assume at some point you will study that. Anyway, you argue that 3-year programs give more cases than 2-year programs, and that does make some sense, obviously. It looked like an average of approximately 100 cases for the extra year. I am not much of a mathematician, but therefore I would have a 10-year program and get 1000 more cases. How do you pick where you stop?

Dr Prasad. The challenge of writing anything, and I think the challenge of the Board, is do case numbers mean anything? That is one of the few things we have that are objective. We have case numbers and pass rates. And whether somebody takes 5 or 500 cases to perform a CABG, I can't tell you, and I don't have that data. I can only use the data that were provided to me and that are actually captured by the Board. What is important is that there is a discrepancy in the programs from $2 \mathrm{Y} 1 \mathrm{R}$ to $3 \mathrm{Y} 3 \mathrm{R}$ and that everybody sits for the same certification, and that dilutes the quality we show everybody else in the real world. One of the points of the article is to have people open their eyes and throw ideas out that we need to change, maybe consolidate, diversify the cases but consolidate the training, and that is really the point.

Dr Kron. Finally, you mentioned appropriately that the exam pass rate is way down, particularly this year, $86 \%$ to $71 \%$. I believe, if I have this right, this is the first year that they examined folks who didn't have to pass the general surgery board. That was a big hurdle for people. Couldn't that be an alternative explanation for the major decrease in pass rate?

Dr Prasad. Absolutely, Dr Kron, although I think that some of the work you have done previously by separating the thoracic boards from the American Board of Surgery will probably be what will save our fellowship. It could definitely be that the people did not sit for the ABS. I don't particularly have an explanation. That was only significant last year, and it could be. It does correlate with the first decrease of applicants, who were 1 to 1 for a long time. So it could be multifactorial. But the point of that slide is there is a change.

Dr Kron. Dr Prasad, I think you did a good job of putting this together. The major issue is that I don't think the time spent in training is equivalent to competency. The question is whether we can measure competency in other ways. There are going to be many ways to train residents, and we just have to study them. The new 6-year models are out there. We are going to have a lot of ways of doing this. I would suggest the years spent may not be as important as the individual experience.

Dr Craig Miller (Stanford, Calif). Let me throw that back to you, Irv. Number one, Dr Prasad, did you or did you not know how many of the people taking the exam last year had ABS certification?

Dr Prasad. No, I did not. It was not provided. 
Dr Miller. Thank you. Let me turn the ball over to the chairman of the RRC-TS. Irv, what are you going to do about this?

Dr Kron. Well, actually, Bill Baumgartner and I spoke last night, and we are going to study that particular question. It was a hurdle. Now, we had to separate this under Fred Crawford's leadership. General surgery certification was separated from thoracic. That allowed for more flexibility, but you never know what you lose by doing that, and that, I believe, may be the major reason for this particular change.

Dr Miller. Is there going to be any pressure to reduce the number of 2-year programs?

Dr Kron. Well, the residency review committee can't work in that way. It purely works at looking at what people do in a given residency. But as you are about to announce, there is going to be a new change in the way we look at thoracic surgical education, and that may be the answer.

Dr Alexander S. Geha (Rancho Santa Fe, Calif). Dr Prasad, I enjoyed this article. I congratulate you and your coauthors for the work that you put into it. I think it is important to focus our attention on it and this aspect in the training programs. There is no question, as Dr Kron said, that the specialty is becoming less attractive to candidates, medical students, surgical residents, and so forth, as shown by the significant decrease in applicant rate, applicant numbers, and filled programs.

The issue is an important one that our specialty and its societies and the Board of Thoracic Surgery and the Residency Review Committee have to address. At issue is the relevance of what we do, and I think you touched on the relevance of what we do and teach in our training programs compared with the reality of what we are called on to do nowadays. The specialty is in a great deal of flux and change, and I think we have to keep up with that and keep our training programs relevant. The leadership in thoracic surgery is already looking into restructuring and reshaping cardiothoracic surgical residency education to meet these challenges and keep up with this reality. Otherwise, to use Dr Prasad's analogy, we may be tailoring beautiful short pants for a black tie gala affair, which would look extremely odd and certainly inappropriately out of place.

I have 2 questions for Dr Prasad. I would like him to comment on whether he thought he was prepared for practice when he finished his, I take it, 3-year residency program, and if he thought there were any changes that should be introduced into his program and other training programs and what those changes would be.

Dr Prasad. I actually graduated from a 2-year program. The offset is that my training started a long time ago when I was a cardiothoracic tech for Dr Tom Murphy at 16 years of age, and I did that for 2 years when I was in high school. Then I went into Dr Brad Allen's lab when I was a second-year medical student for a whole year and did Dr Buckberg's cardioplegia model. Then when I went to Wash U to interview, I interviewed with Dr Bill Gay, and when I was a second-year surgery resident, I was there with Michael Pasque and Rick Barner, and Dr Sundt may not remember this, but he let me do a sternotomy as a second-year resident and let me sew in a proximal, and these are things that I remember.

Then I went into the lab with Drs Damiano and Moon for 2 years, and I experimented on probably 200 animals. After that I spent 4 months on the thoracic service with Drs Cooper and Patterson, and then my 2 years started in cardiothoracic training. So I think
I am well prepared, but I had phenomenal role models, and every time I present and every time I go, I reflect on my mentors, and that is why I think I am well prepared.

Dr Geha. That is a 10-year program according to Dr Kron. You have already put in at least 10 years by my count.

Dr Prasad. Yes, sir. And the only thing that hasn't prepared me was that where I trained, everything came to one institution, and I went to Chicago, which has 58 cardiothoracic open heart programs in a 20-mile radius. I went to one institution and now I go to 4 , and I operate in 3 . But the biggest change has been I operate and then I leave, and I go operate somewhere else and I have to follow all these patients versus one center.

Dr Craig Miller (Stanford, Calif). I congratulate your mentors for opening your eyes, and it sounds like 10 years with Dave Sabiston almost, doesn't it?

Dr Prasad. Absolutely. It was 10 years with the Wash U family.

Dr Chris Tchervenkov (Montreal, Canada). In 2006 in Philadelphia the President of this Association, Richard Jonas, talked about the globalization of cardiac surgery, risks, rewards, and responsibilities. From your data it appears that an increasing number of programs are not matched, up to $40 \%$. Should any consideration be given not to lose this valuable training experience and offer it to train the rest of the world so that these people can get fantastic training in the United States and then return to their countries and help their countries in this exciting field? And what would the obstacles be to such an approach, Dr Kron? Are there any political, organizational obstacles to consider the possibility of training people for the rest of the world?

Dr Prasad. To answer the questions about training foreign graduates on a US model, one of the things we have to look at is that the US model is an ACGME model, and the role of the ACGME is to train US medical graduates; it is funded by the US system. So from a point of quality, although this reflects nothing on the clinicians whatsoever, but from an administrative standpoint, matching with US graduates is thought to be of a higher caliber for a US training program. The US graduates fill approximately $50 \%$ of the spots. So foreign graduates fill the rest of the spots. The question I would leave to Dr Kron, who knows this better than I do, would be funding, because if you match through the match, then you are funded through the ACGME for either 2 or 3 years depending on what your accreditation is. I will defer to Dr Kron about having more foreign graduates in these programs.

Dr Ara Vaporciyan, MD (Houston, Tex). When you looked at the trends over those 6 years, there was clearly some self-selection by the residents of the programs they were choosing. Did you analyze that factor? Did the types of residencies that were being excluded by the applicants have an effect on the case volume?

Dr Prasad. That is a good point, but it is an open market for resident application. The match is obviously blinded to both sides. An important point is that residents and applicants go where they think they will receive better training and can consolidate programs. I think that is important. Of course, programs that have better names, better outcomes, and postgraduate work are going to attract better people, and I think that is a self-selection bias. I don't know a way to control for that because it is an open market, and the market should, as it is doing right now, select out the good programs, and the programs that don't fill will probably be the less-competitive programs. 


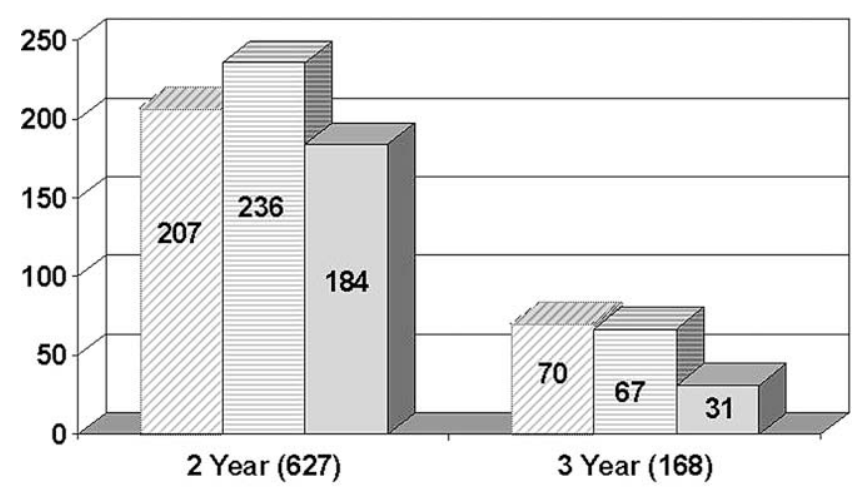

$\square 1$ Resident $\boxminus 2$ Residents $\square 3$ Residents

Figure E1. Resident profile 2002-2007. 